\title{
A founder mutation of CANT1 common in Korean and Japanese Desbuquois dysplasia
}

\author{
Jin Dai ${ }^{1,13}$, Ok-Hwa Kim ${ }^{2,13}$, Tae-Joon Cho ${ }^{3}$, Noriko Miyake ${ }^{4}$, Hae-Ryong Song ${ }^{5}$, Tatsuki Karasugi ${ }^{1}$, \\ Satoru Sakazume ${ }^{6}$, Masahide Ikema ${ }^{7}$, Yoshito Matsui ${ }^{8}$, Toshiro Nagai ${ }^{6}$, Naomichi Matsumoto ${ }^{4}$, \\ Hirofumi Ohashi ${ }^{9}$, Naoyuki Kamatani ${ }^{10}$, Gen Nishimura ${ }^{11}$, Tatsuya Furuichi ${ }^{1,12}$, Atsushi Takahashi ${ }^{10}$ \\ and Shiro Ikegawa ${ }^{1}$
}

Desbuquois dysplasia (DBQD) is a severe skeletal dysplasia of autosomal recessive inheritance. DBQD is classified into types 1 and 2 based on presence or absence of hand anomalies. In a previous study, we found a CANT1 (for calcium-activated nucleotidase 1) mutation, c.676G $>$ A in five DBQD families. They were all East Asians (Japanese or Korean). The high prevalence of the same mutation among Japanese and Korean suggested that it is a common founder mutation in the two populations. To examine a possible common founder, we examined the region around CANT1 in chromosomes with c.676G $>A$ mutation by genotyping polymorphic markers in the region for the families. We examined their haplotypes using the family data. We identified in all families a common haplotype containing the CANT1 mutation that ranged up to $550 \mathrm{~kb}$. The two unrelated carriers of the mutation in general populations in Korea and Japan could also have the haplotype. We estimated the age of the founder mutation as $\sim 1420$ years $(95 \% \mathrm{Cl}=880-1940$ years). The c.676G $>$ A mutation of $C A N T 1$ commonly seen in Japanese and Korean DBQD should be derived from a common founder.

Journal of Human Genetics (2011) 56, 398-400; doi:10.1038/jhg.2011.28; published online 17 March 2011

Keywords: calcium-activated nucleotidase 1; common founder; common haplotype; desbuquois dysplasia

\section{INTRODUCTION}

Desbuquois dysplasia (DBQD; MIM \#251450) is a severe skeletal dysplasia of autosomal recessive inheritance that belongs to 'multiple dislocation group. ${ }^{1}$ DBQD is clinically heterogeneous and classified into two types on the basis of presence (type 1) or absence (type 2) of characteristic hand anomalies, which consist of an extra ossification center distal to the second metacarpal, delta phalanx, bifid distal thumb phalanx and dislocation of the inter-phalangeal joints. ${ }^{2}$ Type 2 DBQD contained a further clinical subtype, Kim variant, ${ }^{3}$ which is characterized by short metacarpals with elongated phalanges that result in nearly normal length of the fingers.

Recently, Huber et al. ${ }^{4}$ identified mutations in the gene encoding the calcium-activated nucleotidase 1 (CANT1) in DBQD type 1, which is followed by Faden et al. ${ }^{5}$ We also found CANT1 mutations in DBQD type 2 and Kim variant. In our series, we identified CANT1 mutations in all seven patients from five unrelated families with DBQD Kim variant, and all patients had c.676G > A (p.V226M).
The five families were Japanese or Korean. c.676G $>$ A was also found in 1/754 Japanese and 1/187 Korean controls in a heterozygous state. ${ }^{6}$ These results led us consider that the mutation may be inherited from a common founder(s) among Japanese and Korean. To test the hypotheisis, we examined haplotypes of the patients around CANT1 and found that the mutation was on a common haplotype background of $\sim 500 \mathrm{~kb}$.

\section{MATERIALS AND METHODS}

Subjects

Seven DBQD patients from five families (four Japanese and three Korean) and their parents were examined. One Japanese family (DB1) was consanguineous (1st cousin marriage) and two sib patients from the family had c.676G $>$ A in a homozygous state. The other patients were of non-consanguineous and compound heterozygotes of the mutations. ${ }^{6}$ Their phenotypes were all diagnosed as DBQD Kim variant as previously reported. ${ }^{3}$ One Japanese (CT1) and one Korean (CT2) subject from general populations who were found to have c.676G $>\mathrm{A}$ in a heterozygous state in the previous study were also examined. ${ }^{3}$

\footnotetext{
${ }^{1}$ Laboratory of Bone and Joint Diseases, Center for Genomic Medicine, RIKEN, Tokyo, Japan; ${ }^{2}$ Department of Radiology, Ajou University Hospital, Suwon, Korea; ${ }^{3}$ Department of Orthopaedic Surgery, Seoul National University Children's Hospital, Seoul, Korea; ${ }^{4}$ Department of Human Genetics, Yokohama City University Graduate School of Medicine, Yokohama, Japan; ${ }^{5}$ Department of Orthopedic Surgery, Korea University Guro Hospital, Seoul, Korea; ${ }^{6}$ Department of Pediatrics, Dokkyo Medical University Koshigaya Hospital, Koshigaya, Japan; ${ }^{7}$ Department of Orthopaedic, Nagasaki Prefectural Center of Medicine and Welfare for Children, Nagasaki, Japan; ${ }^{8}$ Department of Orthopedic Surgery, University of Toyama, Toyama, Japan; ${ }^{9}$ Division of Medical Genetics, Saitama Children's Medical Center, Iwatsuki, Japan; ${ }^{10}$ Laboratory for Statistical Analysis, Center for Genomic Medicine, RIKEN, Tokyo, Japan; ${ }^{11}$ Department of Pediatric Imaging, Tokyo Metropolitan Children's Medical Center, Fuchu, Japan and ${ }^{12}$ Laboratory Animal Facility, Research Center for Medical Sciences, Jikei University School of Medicine, Tokyo, Japan

${ }^{13}$ These authors are contributed equally to this work.

Correspondence: Dr S Ikegawa, Laboratory of Bone and Joint Diseases, Center for Genomic Medicine, RIKEN, 4-6-1 Shirokanedai, Minato-ku, Tokyo 108-8639, Japan. E-mail: sikegawa@ims.u-tokyo.ac.jp
}

Received 15 December 2010; revised 26 January 2011; accepted 16 February 2011; published online 17 March 2011 


\section{Genotyping}

Genomic DNA was extracted from blood by standard procedures or from saliva using Oragene DNA Self-Collection kit (DNA Genotek, Ottawa, Ontario, Canada). Affymetrix 10K Gene-Chip microarray (Affymetrix, Santa Clara, CA, USA) was used for family DB1 to determine homozygous regions containing CANT1 inherited from a common ancestor in the consanguineous family. Single nucleotide polymorphisms (SNPs) for the founder haplotype determination were genotyped by direct sequencing. The regions containing targeted SNPs were amplified by PCR from genomic DNA, and then PCR products were diluted and sequenced by using an ABI Prism 3730 automated sequencer (PE Biosystems, Tokyo, Japan). We separated 5'-FAM-labeled PCR products containing microsatellites by sizes on an ABI Prism 3730 automated sequencer (PE Biosystems) against the Genescan-500LIZ size standard. PCR primers for the polymorphisms are listed in Supplementary Table 1.

\section{Haplotype analysis}

We determined the mutation-containing haplotype of patients according to the genotypes of the trio (the patient and parents). We determined the homozygous region of DB1 using the microarray data of the family members. All the patients were genotyped for the markers selected from the homozygous region of DB1. The genotyping of the makers for their parents were examined only when the patients were heterozygotes. The boundaries of the common haplotype were determined by sequential genotyping of SNPs in flanking regions. The SNPs were selected from the HapMap database (Phase II+ III, release 27).

\section{Estimation of the mutation age}

We estimated the age of the founder mutation by the method previously described. ${ }^{7,8}$ We used the following equation:

$$
g=\log \left[\left(p_{d}-p_{n}\right) /\left(1-p_{n}\right)\right] / \log (1-\theta),
$$

where $p_{d}$ and $p_{n}$ denote frequencies of the ancestral allele at the marker locus on the chromosomes with $A$ and $G$ alleles at the disease locus. $\theta$ denotes the recombination fraction between the marker and the disease locus. The age of the mutation was corrected by taking the population growth into account using the following equations:

$$
\begin{aligned}
& g_{c}=g+g_{0}, \\
& g_{0}=-(1 / d) \ln \left[\theta \times e^{d} /\left(e^{d}-1\right)\right],
\end{aligned}
$$

where $d$ denotes the population growth rate. Physical and genetic map positions in bp and cM for SNP loci were obtained from the HapMap database (Phase II, release 21).

\section{RESULTS}

\section{Genotyping and identification of a common haplotype}

Using the SNP microarray data, we first determined a homozygous region of the genome that contained CANT1 in the consanguineous family, DB1. The family had a stretch of SNPs that was homozygous in the patients and heterozygous in the parents. The homozygous stretch flanked by rs2934226 and rs2306755 extended up to $\sim 9.8 \mathrm{Mb}$. Of 48 SNPs that composed the stretch in the microarray data, we selected 10 SNPs around CANT1; six were at the $3^{\prime}$ to CANT1 and four were at the $5^{\prime}$ to CANT1. We genotyped them for the five families. A possible common haplotype was restricted in the region between rs6501224 and rs2377402.

To confirm the common haplotype and define its more detailed boundary, we selected 13 SNPs in the boundary regions from a database and genotyped them for the five families. In the SNPs we could get a definite haplotype result, all in a region from rs10512617 to rs2045660 showed a common haplotype. The nearest SNPs to rs10512617 and rs2045660 that defined the different haplotypes in any of the five families were rs11077391 and rs2377309, respectively. At the SNPs between

\begin{tabular}{|c|c|c|c|c|c|c|c|c|}
\hline \multirow[b]{3}{*}{ Marker } & \multirow[b]{3}{*}{ Distance $e^{\mathrm{a}}$} & \multicolumn{7}{|c|}{ Subject } \\
\hline & & \multicolumn{5}{|c|}{$D B Q D$ Family } & \multicolumn{2}{|c|}{ Control } \\
\hline & & $D B 1$ & $D B 2$ & $D B 3$ & $D B 4$ & $D B 5$ & CT1 & CT2 \\
\hline rs7405591 & 1712736 & $\mathrm{C}$ & $\mathrm{T}$ & $\mathrm{T}$ & $\mathrm{T}$ & C & $\mathrm{T}$ & $\mathrm{T}$ \\
\hline rs4789523 & 978405 & $\mathrm{~T}$ & $\mathrm{~T}$ & $\mathrm{~T} / \mathrm{C}^{\mathrm{b}}$ & $\mathrm{T}$ & $\mathrm{T}$ & $\mathrm{T} / \mathrm{C}$ & $\mathrm{T} / \mathrm{C}$ \\
\hline rs4129767 & 587275 & C & C & C & C & $\mathrm{T}$ & C & $\mathrm{C} / \mathrm{T}$ \\
\hline rs6501224 & 488804 & G & $G$ & $\mathrm{C}$ & G & G & G & G \\
\hline rs16971539 & 434643 & A & $G$ & $G / A^{b}$ & G & G & G & $G$ \\
\hline rs9899295 & 402575 & A & $G$ & A & G & G & $A / G$ & $G$ \\
\hline rs9896451 & 347233 & G & $G$ & $A$ & G & $G$ & $G$ & $\mathrm{G} / \mathrm{A}$ \\
\hline rs11077391 & 330052 & $G$ & $A$ & $A$ & G & $A$ & $\mathrm{G}$ & $G$ \\
\hline rs10512617 & 297708 & G & $G$ & G & G & $G$ & $\mathrm{G}$ & $G$ \\
\hline rs4103047 & 254069 & C & C & $\mathrm{C}$ & C & C & C & $C / G$ \\
\hline rs3744801 & 197531 & $\mathrm{C}$ & $\mathrm{C}$ & C & $\mathrm{C}$ & C & $\mathrm{C} / \mathrm{T}$ & $\mathrm{C}$ \\
\hline rs2889529 & 93784 & C & $\mathrm{C}$ & C & $\mathrm{C}$ & C & $\mathrm{C} / \mathrm{T}$ & $\mathrm{C} / \mathrm{T}$ \\
\hline c. $676 \mathrm{G}>\mathrm{A}$ & 0 & & & & & & & \\
\hline rs8077024 & 2287 & $\mathrm{C}$ & C & $\mathrm{C}$ & C & C & $\mathrm{C} / \mathrm{T}$ & $\mathrm{C}$ \\
\hline D17S1847 & 33602 & 185 & 185 & 185 & 185 & 185 & 185/191 & $185 / 189$ \\
\hline rs2707047 & 132355 & $\mathrm{G}$ & $G$ & G & G & G & $\mathrm{G} / \mathrm{A}$ & $G / A$ \\
\hline rs2612787 & 132449 & $\mathrm{~T}$ & $\mathrm{~T}$ & $\mathrm{~T}$ & $\mathrm{~T}$ & $\mathrm{~T}$ & $\mathrm{~T} / \mathrm{C}$ & $\mathrm{T} / \mathrm{C}$ \\
\hline rs9302889 & 168892 & $\mathrm{~T}$ & $\mathrm{~T}$ & $\mathrm{~T}$ & $\mathrm{~T}$ & $\mathrm{~T}$ & $\mathrm{~T} / \mathrm{A}$ & $\mathrm{T}$ \\
\hline rs12600665 & 180703 & $G$ & G & G & G & G & $G / A$ & G \\
\hline rs2045660 & 185092 & C & C & C & C & C & C & C \\
\hline rs2377309 & 201821 & C & C & C & C & $\mathrm{C} / \mathrm{T}^{\mathrm{b}}$ & C & $\mathrm{C} / \mathrm{T}$ \\
\hline rs2612753 & 220795 & $G$ & G & $G$ & G & $\mathrm{T}$ & $\mathrm{G} / \mathrm{T}$ & $G$ \\
\hline rs1000791 & 274619 & $\mathrm{~T}$ & $A$ & $\mathrm{~T}$ & $\mathrm{~T}$ & $\mathrm{~T}$ & $\mathrm{~T}$ & $\mathrm{~T} / \mathrm{C}$ \\
\hline rs2377402 & 340187 & $A$ & C & $\mathrm{C}$ & A & C & C & C \\
\hline
\end{tabular}

Table 1 Haplotypes of markers with the $\mathrm{c} .676 \mathrm{G}>\mathrm{A}$ mutation in the Desbuquois dysplasia (DBQD) families and control subjects

aBase number between the marker and the disease locus.

${ }^{b}$ Equivocal because of the double heterozygosity of the parents.

rs10512617 and rs2045660, the two carriers of the mutation (CT1 and CT2) had all the alleles that composed the common haplotype. Therefore, they could also have the same haplotype (Table 1).

\section{Estimation of the mutation age}

The markers that defined the haplotype of the families were used for estimation of the mutation age. The disease chromosomes of DB1 were counted as one because of its consanguinity. $p_{d}$ was calculated in five chromosomes with A allele at the disease locus from the five DBQD families. As frequency of the mutation (A allele) was quite low in control subjects, we regarded the allele frequency of markers in the general Japanese population as $p_{n}$, and the allele frequencies were obtained from NCBI dbSNP database. The population growth rate, $\mathrm{d}$ was set to 0.08 as previously described. ${ }^{7}$ The allele, frequency of which was higher in the five chromosomes than that in the general population was regarded as the ancestral allele. We excluded the markers in the region less than $60 \mathrm{~kb}$ from the disease locus. ${ }^{9}$ The estimated ages by different markers ranged from 30 to 246 generations (mean $=71$; 95\% CI $=44-97$; median $=41$; standard deviation $=58$ ) (Table 2). If we assumed an intergenerational time of 20 years, the age of the mutation was estimated to be 1420 years (95\% CI $=880-1940$ years; median $=820$ years; standard deviation $=1160$ years $)$.

\section{DISCUSSION}

We have demonstrated that the $.676 \mathrm{G}>\mathrm{A}$ mutation in Japanese and Korean had a common founder because all five chromosomes with 
Table 2 Estimated age of the c.676G $>$ A mutation

\begin{tabular}{lcccccr}
\hline Marker & $P d$ & $P n$ & $\theta$ & $g$ & $g 0$ & \multicolumn{1}{c}{$g c$} \\
\hline rs7405591 & 0.4 & 0.295 & 0.0545 & 34.0 & 4.3 & 38.3 \\
rs4129767 & 0.8 & 0.488 & 0.0191 & 25.7 & 17.4 & 43.1 \\
rs6501224 & 0.8 & 0.698 & 0.0145 & 74.5 & 20.9 & 95.4 \\
rs9899295 & 0.6 & 0.430 & 0.00998 & 120.6 & 25.5 & 146.1 \\
rs9896451 & 0.8 & 0.488 & 0.00432 & 114.3 & 36.0 & 150.3 \\
rs11077391 & 0.6 & 0.295 & 0.00401 & 208.6 & 37.0 & 245.6 \\
rs10512617 & 1 & 0.400 & 0.00394 & 0 & 37.1 & 37.1 \\
rs4103047 & 1 & 0.659 & 0.00391 & 0 & 37.2 & 37.2 \\
rs3744801 & 1 & 0.523 & 0.00385 & 0 & 37.4 & 37.4 \\
rs2889529 & 1 & 0.233 & 0.00187 & 0 & 46.5 & 46.5 \\
c.676G $>$ A & & & 0 & & & \\
rs2707047 & 1 & 0.145 & 0.00407 & 0 & 36.7 & 36.7 \\
rs2612787 & 1 & 0.024 & 0.00408 & 0 & 36.7 & 36.7 \\
rs9302889 & 1 & 0.727 & 0.00691 & 0 & 30.1 & 30.1 \\
rs12600665 & 1 & 0.500 & 0.00722 & 0 & 29.6 & 29.6 \\
rs2045660 & 1 & 0.576 & 0.00727 & 0 & 29.5 & 29.5 \\
rs2612753 & 0.8 & 0.589 & 0.0106 & 62.8 & 24.8 & 87.6 \\
rs1000791 & 0.8 & 0.625 & 0.0159 & 47.6 & 19.7 & 67.3 \\
rs2377402 & 0.4 & 0.157 & 0.0201 & 61.3 & 16.8 & 78.1 \\
\hline
\end{tabular}

c.676A in DBQD patients had a common haplotype composed of nine markers around this disease locus. In addition, the genotype of the two unrelated normal subjects with this mutation contained all the alleles that composed the common haplotype, suggesting that the founder mutation has widely spread among Korean and Japanese populations. The haplotype region ranged $480-550 \mathrm{~kb}$. We estimated the age of this mutation at $\sim 1400$ years $(95 \% \mathrm{CI}=880-1940$ years). Although the calculated $\mathrm{CI}$ is quite wide, we can estimate the c.676G $>$ A mutation dates back to a time around the late Kofun era. Because the allele frequency of c.676G $>\mathrm{A}$ in Korean controls is much higher than that in Japanese controls, we speculated that the common founder was a Korean, and this mutation spread from Korea to Japan. This speculation is not contradictory to migration from Korea to Japan in the ancient history.

Although the exact prevalence of DBQD is difficult to determine, DBQD is generally considered as a very rare disease. However, the carrier frequency of the common mutation is considerably high in Korean. From our experience, ${ }^{6}$ the frequency of the CANT1 mutation is speculated as about double of that of the common mutation. Thereafter, prevalence of DBQD is $\sim 1 / 35000$ in Korean and $\sim 1 / 600000$ in Japanese; the former is far higher than our impression in daily practice. Many DBQD patients caused by CANT1 mutations may be pre- or peri-natally lethal, although all the East Asian cases of DBQD with CANT1 mutations so far reported had good prognosis. Alternatively, many DBQD may be undiagnosed and/or be put into the waste box of diagnosis as unknown 'multiple dislocation group' of skeletal dysplasia. Diagnosis of DBQD based on clinical and/or radiographic information is sometimes very difficult because of the overlapping phenotype with other diseases. ${ }^{10}$

In DBQD patients, the founder mutation was identified in all the seven Kim variant patients, and only in the Kim variant patients to our knowledge. Therefore, this mutation may be closely related with specific phenotypes of the Kim variant; that is , accelerated carpal bone ages in childhood, short metacarpals, elongated appearance of phalanges and absence of accessory ossification center distal to the second metacarpal and thumb anomalies. ${ }^{3}$ Further accumulation of Korean and Japanese DBQD patients and examination of their CANT1 mutations are necessary to conclude relation of the founder mutation and the specific phenotype. The founder mutation may present with other phenotype. Moreover, further collection of DBQD patients and screening of CANT1 mutations in other populations would help confirming the phenotype-genotype relation. Other mutation (s) may present with the Kim-type DBQD phenotype. The diagnosis of DBQD is sometimes very difficult at present; however, we think identification of the specific phenotypes of hands followed by examination of the founder mutation in CANT1 would efficiently led us to definite diagnosis in Japanese and Korean patients suspected for having DBQD.

\section{ACKNOWLEDGEMENTS}

We are grateful to Dr Yoshiyuki Yukawa for helping with statistics work. We are grateful to patients, their family members and their doctors for participating in the study. This project was supported by Grants-in-aids from the Ministry of Education, Culture, Sports and Science of Japan (Contract grant No. 20390408), from Research on Child Health and Development (Contract grant No. 20-S-3), from the Ministry of Health, Labor and Welfare of Japan (Measures for Intractable Diseases 046 in 2010) and from the Ministry for Health, Welfare and Family Affairs of Republic of Korea (Korea Healthcare Technology R\&D Project; Contract grant A080588). This project was also supported by the Japanese Skeletal Dysplasia Consortium.

1 Superti-Furga, A. \& Unger, S. Nosology and classification of genetic skeletal disorders: 2006 revision. Am. J. Med. Genet. A 143, 1-18 (2007).

2 Faivre, L., Cormier-Daire, V., Eliott, A. M., Field, F., Munnich, A., Maroteaux, P. et al. Desbuquois dysplasia, a reevaluation with abnormal and 'normal' hands: radiographic manifestations. Am. J. Med. Genet. A 124A, 48-53 (2004).

3 Kim, O. H., Nishimura, G., Song, H. R., Matsui, Y., Sakazume, S., Yamada, M. et al. A variant of Desbuquois dysplasia characterized by advanced carpal bone age, short metacarpal, and elongated phalanges: report of seven cases. Am. J. Med. Genet. A 152A, 875-885 (2010).

4 Huber, C., Oules, B., Bertoli, M., Chami, M., Fradin, M., Alanay, Y. et al. Identification of CANT1 mutations in Desbuquois dysplasia. Am. J. Hum. Genet. 85, 706-710 (2009).

5 Faden, M., Al-Zahrani, F., Arafah, D. \& Alkuraya, F. S. Mutation of CANT1 causes Desbuquois dysplasia. Am. J. Med. Genet. A 152A, 1157-1160 (2010).

6 Furuichi, T., Dai, J., Cho, T. J., Sakazume, S., Ikema, M., Matsui, Y. et al. CANT1 mutation is also responsible for Desbuquois dysplasia, type 2 and Kim variant. J. Med. Genet. 48, 32-37 (2011).

7 Ichida, K., Hosoyamada, M., Kamatani, N., Kamitsuji, S., Hisatome, I., Shibasaki, T. et al. Age and origin of the G774A mutation in SLC22A12 causing renal hypouricemia in Japanese. Clin. Genet. 74, 243-251 (2008).

8 Risch, N., de Leon, D., Ozelius, L., Kramer, P., Almasy, L., Singer, B. et al. Genetic analysis of idiopathic torsion dystonia in Ashkenazi Jews and their recent descent from a small founder population. Nat. Genet. 9, 152-159 (1995).

9 Jorde, L. B., Watkins, W. S., Carlson, M., Groden, J., Albertsen, H., Thliveris, A. et al. Linkage disequilibrium predicts physical distance in the adenomatous polyposis coli region. Am. J. Hum. Genet. 54, 884-898 (1994).

10 Miyake, A., Nishimura, G., Futami, T., Ohashi, H., Chiba, K., Toyama, Y. et al. A compound heterozygote of novel and recurrent DTDST mutations results in a novel intermediate phenotype of Desbuquois dysplasia, diastrophic dysplasia, and recessive form of multiple epiphyseal dysplasia. J. Hum. Genet. 53, 764-768 (2008).

Supplementary Information accompanies the paper on Journal of Human Genetics website (http://www.nature.com/jhg) 